\title{
The pollution of the interstellar medium from AGB stars in Globular Clusters
}

\author{
Paolo Ventura ${ }^{1}$ and Roberta Carini ${ }^{1,2}$ \\ ${ }^{1}$ INAF - Osservatorio Astronomico di Roma \\ Via Frascati 33, 00040, Monte Porzio Catone (RM), Italy \\ email: paolo.ventura@oa-roma.inaf.it \\ ${ }^{2}$ Universitá di Roma "La Sapienza" \\ Piazzale Aldo Moro 5, 00135 Rome, Italy \\ email: roberta.carini@oa-roma.inaf.it
}

\begin{abstract}
We discuss the yields from Asymptotic Giant Branch stars, depending on their mass and metallicity. In agreement with previous investigations, we find that the extent of Hot Bottom Burning increases with mass. The yields of models with chemistry typical of high-metallicity Globular Clusters, i.e. $\mathrm{Z}=0.008$, show only a modest depletion of magnesium, and an oxgen depletion of $\sim 0.4 \mathrm{dex}$. Low-metallicity yields show a much stronger magnesium depletion, and a dramatic drop in the oxygen content, $\sim 1.2$ dex smaller than the initial value. We suggest that the Globular Cluster NGC 2419 is a possible target to the hypothesis of the self-enrichment scenario of Globular Clusters by the winds of Asymptotic Giant Branch stars.
\end{abstract}

Keywords. stars: AGB and post-AGB, stars: abundances, globular clusters: general

\section{Introduction}

Intermediate mass stars, with mass $M \geqslant 4 M_{\odot}$, have been proposed as the sources favoring the pollution of the interstellar medium in Globular Clusters (GC) (Ventura et al. 2001), giving origin to the formation of further stellar generations. The new stars would form in the environment polluted by the gas ejected by stars in the Asymptotic Giant Branch (AGB) phase, when they loose their external mantle. This gas will present traces of Hot Bottom Burning (HBB), showing up the patterns produced by $\mathrm{p}$-capture nucleosynthesis.

In this contribution we discuss how the yields of massive AGBs change with mass and metallicity, and try to deduce the chemistry of stars of Globular Clusters belonging to the second generation (SG), depending on their metallicity.

\section{Yields from AGBs and SAGBs}

The yields from AGB models are shown in Fig. 1. The metallicities examined are $Z=3 \times 10^{-4}, Z=10^{-3}$, and $Z=8 \times 10^{-3}$, which represent the chemistry of GCs with low, intermediate and high metallicity.

The oxygen and sodium yields are correlated: at large temperatures $(T>70 \mathrm{MK})$ the destruction channel for sodium prevails, so that, while oxygen is consumed, part of the sodium previously accumulated via the second dredge- up and ${ }^{22} \mathrm{Ne}$ burning, is destroyed.

The low-Z models show a more extreme chemistry, i.e. more oxygen-poor; this is a clear indication that at low Z's HBB takes place at larger temperatures, favoring a more advanced nucleosynthesis.

The right panel of Fig. 1 shows the yields of AGB stars in the $\mathrm{Mg}-\mathrm{Al}$ plane. The $Z=$ $3 \times 10^{-4}$ models show-up the greatest depletion of magnesium, because of the higher HBB 


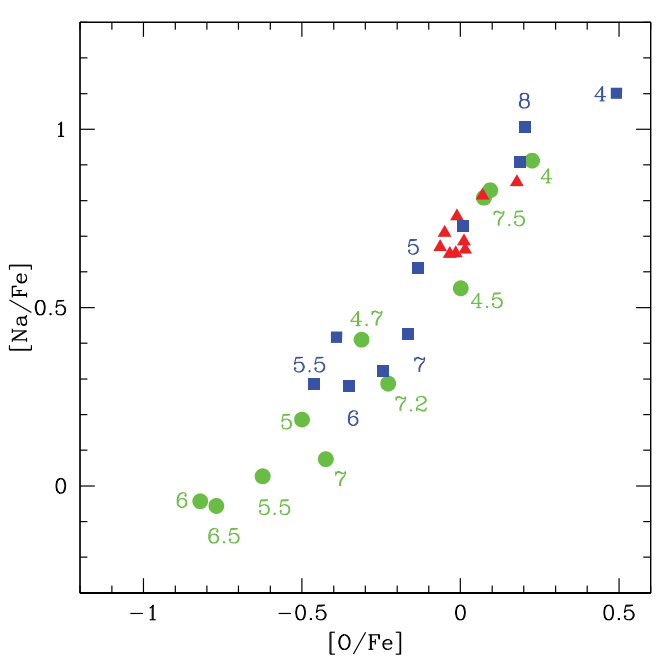

(a)

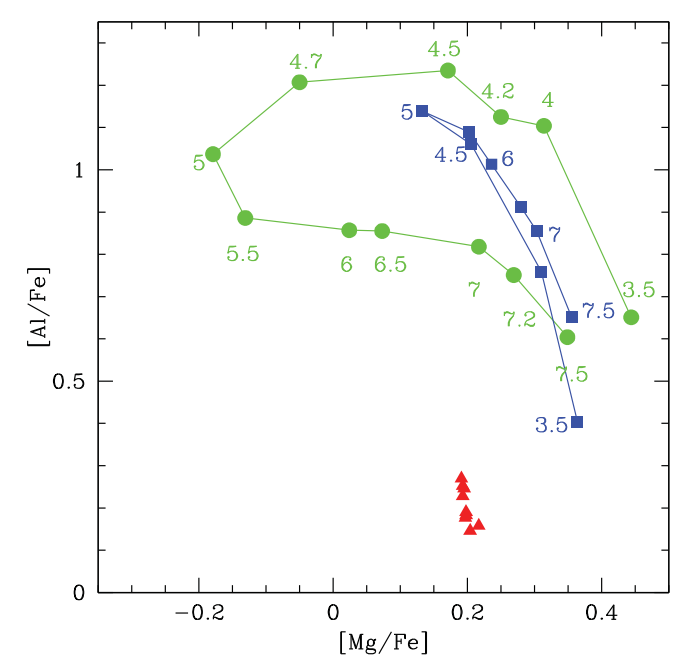

(b)

Figure 1. Yields of AGB stars in the $\mathrm{O}-\mathrm{Na}$ and $\mathrm{Mg}-\mathrm{Al}$ plane. The meaning of symbols is as follows. green points: $Z=3 \times 10^{-4}$; blue squares: $Z=3 \times 10^{-3}$; red triangles: $Z=8 \times 10^{-3}$

temperatures. The maximum Al-enhancement reaches $\sim 1-1.2 \mathrm{dex}$, eventually becoming independent of the $\mathrm{Mg}$-depletion. This finds a motivation in the saturation occurring at very large T's, exceeding $\sim 100 \mathrm{MK}$, when production and destruction channels of Aluminium compensate.

In both panels of Fig. 1 we note that the stars producing the ejecta with the most extreme chemistry are those with masses around $\sim 5.5-6 M_{\odot}$, at the edge between the $\mathrm{AGB}$ and the SAGB regimes. The reason is that despite massive AGBs experience a stronger $\mathrm{HBB}$, they loose mass with a higher rate: they loose their envelope before a great modification of the surface chemistry may occur (Ventura \& D'Antona 2011).

\section{The implications for the self-enrichment of Globular Clusters}

Based on the results discussed above, we predict that low-metallicity clusters with a "pure" second generation (i.e. formed directly from the wind of AGB stars) should show the signature of a very advanced p-capture nucleosynthesis, with extremely Opoor chemistry. Sodium increase of these stars is also predicted to be modest.

NGC 2419 could be the ideal target to test these predictions: the Horizontal Branch of this cluster is known to harbor a group of blue stars separated from the main component (Ripepi et al. 2007), that, in analogy with the case of NGC 2808, is likely to be composed by a pure SG, made up of helium-rich stars.

Spectroscopic analysis of the the Main sequence counterparts of these stars could help accepting or disregarding the self-enrichment scenario hypothesis.

\section{References}

Ripepi, V., et al. 2007, ApJ, 667, L61

Ventura, P., D’Antona, F., Mazzitelli, I., \& Decressin, T. 2001, ApJ, 550, L65

Ventura, P. \& D'Antona, F. 2011, MNRAS, 410, 2760 